\title{
Modélisation des avalanches denses Approches théorique et numérique
}

\author{
Christophe Ancey \\ Centre d'Etudes du Machinisme Agricole, du Génie Rural des Eaux et Forêts \\ 2 rue de la Papeterie, 38402 Saint Martin d'Hères, BP 76, tél. : 76762727
}

\section{Introduction}

Au cours du $\mathrm{XX}^{\mathrm{e}}$ siècle, les zones de montagne ont connu une rapide augmentation de leur fréquentation essentiellement liée au développement très important du tourisme et de I'intensification du transport routier ; malheureusement, cette humanisation croissante de la montagne concerne souvent des zones sensibles soumises en hiver et au printemps aux avalanches. Celles-ci provoquent annuellement de nombreuses pertes matérielles voire parfois humaines, même si l'avalanche demeure l'une des catastrophes naturelles les moins destructrices. Le souci d'assurer la sécurité des zones habitées et des infrastructures routières, de garantir la circulation routière quotidiennement, quelles que soient les conditions climatiques, a exigé et exige encore une connaissance accrue du phénomène avalancheux.

Se prémunir contre les avalanches nécessite de délimiter les zones à risques, de construire des ouvrages de protection ou des systèmes de prévention (déclenchement artificiel, signaux d'alerte...) ; il est donc apparu assez tôt intéressant de proposer des modèles susceptibles de décrire la neige et son évolution, que cela soit au repos ou en écoulement. La complexité et la diversité du phénomène rend cette étude très ardue et si de nombreuses modélisations ont été proposées, les résultats ne sont pas toujours satisfaisants. Ce constat a amené la division Nivologie du CEMAGREF à développer de nouveaux modèles pouvant décrire dans ses grandes lignes le phénomène avalancheux. Nous présenterons ici la partie consacrée aux avalanches denses.
Auparavant il ne nous paraît pas inutile de préciser la terminologie employée. Une avalanche est un écoulement gravitaire rapide de neige. C'est une définition très simple, mais une définition plus précise nécessite d'introduire les facteurs morphologiques et génétiques caractérisant les différentes zones affectées par l'écoulement et la qualité de la neige. Nous supposerons tout au long de cet article qu'une avalanche est du point de vue dynamique entièrement caractérisée par son mode d'écoulement; à cet effet, on distinguera [Unesco, 1981] :

- l'avalanche en aérosol (airborne powder avalanche) : c'est un écoulement très rapide (la vitesse peut dépasser les $400 \mathrm{~km} / \mathrm{h}$ ) composé de grandes bouffées turbulentes résultant du mélange des particules de neige avec l'air,

- l'avalanche dense (flowing avalanche, Fliesslawine): c'est un écoulement de neige coulant le long du sol en suivant le relief, avec une vitesse moindre (qui ne dépassera guère les $80 \mathrm{~km} / \mathrm{h}$ ),

- l'avalanche mixte: c'est une avalanche possédant les deux caractéristiques; 1 'aérosol peut se développer et devenir indépendant de l'écoulement dense tandis que dans d'autres cas ces deux écoulements sont intimement liés. Si l'aérosol ne contribue que faiblement à la dynamique de l'ensemble, on parlera d'avalanche dense (et réciproquement).

Nous supposons que cette classification arbitraire est suffisamment pertinente pour classer les différents comportements dynamiques d'une avalanche. Par la suite, on s'intéressera uniquement à la modélisation des avalanches denses,

\section{Modelling flowing avalanches}

This paper focuses on physical and numerical modelling of flowing avalanches. The first part of this investigation examines previous models : it is shown that our sound knowledge of avalanches are insufficient, be it through the rheology of snow or through the accuracy of models. The second part presents a mathematical model based on mass and momentum equations. As constitutive relation, we use a Bingham law. Equations are written in curvilinear coordinates for one-dimensional simply sheared flow. For transient laminar regime, wall friction is calculated using the formula of corresponding uniform steady regime. The numerical resolution of this hyperbolic system is performed by a Godunov's scheme. Numerical simulations show that density variations during flow have no influence on large avalanches and that the determination of yield stress is essential for a correct velocity estimation. 


\section{Notations}

\begin{tabular}{|c|c|c|}
\hline Symbole & Nom & Unité \\
\hline$C$ & cohésion & $\mathrm{Pa}$ \\
\hline$C$ & courbure $C=1 / R$ & $m^{-1}$ \\
\hline$C_{f}$ & courbe de la ligne de pente & \\
\hline$f$ & coefficient de frottement & \\
\hline$F_{f}$ & force de frottement & $N$ \\
\hline$g$ & constante de gravitation $g=9,81$ & $\mathrm{~m} / \mathrm{s}^{2}$ \\
\hline $\mathrm{Fr}$ & $\begin{array}{l}\text { nombre de Froude, } \\
\mathrm{Fr}=\bar{u} / \sqrt{g h \cos \theta}\end{array}$ & \\
\hline $\mathrm{He}$ & $\begin{array}{l}\text { nombre de Hedstrom, } \\
\mathrm{He}=\tau_{c} h^{2} \rho / \mu^{2}\end{array}$ & \\
\hline$H, h$ & hauteur d'écoulement & $\mathrm{m}$ \\
\hline$L$ & longueur de l'avalanche & \\
\hline M & masse de l'avalanche & $\mathrm{kg}$ \\
\hline$r$ & coefficient de frottement solide & \\
\hline$R$ & rayon de courbure de $C f$ & $\mathrm{~m}$ \\
\hline $\operatorname{Re}$ & $\begin{array}{l}\text { nombre de Reynolds } \\
\operatorname{Re}=\rho h u / \mu\end{array}$ & \\
\hline$R_{H}$ & rayon hydraulique & $\mathrm{m}$ \\
\hline$u, v$ & vitesse & $\mathrm{m} / \mathrm{s}$ \\
\hline$x, y$ & axes de coordonnées & $\mathrm{m}$ \\
\hline$\kappa_{\text {actpass }}$ & $\begin{array}{l}\text { coefficient de pression (butée } \\
\text { ou poussée) } \\
\kappa\left\{\begin{array}{c}\text { act } \\
\text { pass }\end{array}=\right. \\
2 \frac{1 \mp \sqrt{1-\left(1+\tan ^{2} \delta\right) \cos ^{2} \phi}-1}{\cos ^{2 \phi}}\end{array}$ & \\
\hline$\alpha$ & paramètre de forme & \\
\hline$x$ & périmètre mouillé & $\mathrm{m}$ \\
\hline$\delta$ & angle de frottement du fond & degré \\
\hline r & nombre d'aspect $\varepsilon=H / L$ & \\
\hline$\eta$ & $\eta=h / h_{c}$ & \\
\hline$\lambda$ & coefficient de courbure $\lambda=L / R$ & \\
\hline
\end{tabular}

\begin{tabular}{|c|c|c|}
\hline$P$ & pression & $P_{a}$ \\
\hline 1 & tenseur unitaire & \\
\hline$\tau_{c}$ & seuil de contrainte & $P_{a}$ \\
\hline$\mu$ & viscosité dynamique & $\mathrm{kgm} / \mathrm{s}^{2}$ \\
\hline$v$ & viscosité cinématique & $\mathrm{m} / \mathrm{s}^{2}$ \\
\hline$\theta$ & angle de pente & rad \\
\hline$\rho$ & masse volumique & $\mathrm{kg} / \mathrm{m}^{3}$ \\
\hline v & contrainte normale & $\mathrm{Pa}$ \\
\hline$\tau$ & contrainte tangentielle & $\mathrm{Pa}$ \\
\hline$\xi$ & $\begin{array}{l}\text { coefficient de frottement tur- } \\
\text { bulent }\end{array}$ & \\
\hline$\xi$ & $\begin{array}{l}\text { rugosité } \xi=h / k_{s}, k_{s} \text { est la } \\
\text { rugosité équivalente }\end{array}$ & $\mathrm{m} / \mathrm{s}^{2}$ \\
\hline$\xi, \eta$ & coordonnées curvilignes & \\
\hline$\underline{\underline{D}}$ & tenseur de déformation & $1 / s$ \\
\hline$\Omega$ & $\begin{array}{l}\text { tenseur antisymétrique de } \\
\text { déformation }\end{array}$ & $1 / s$ \\
\hline$\underline{\underline{T}}$ & tenseur des contraintes & $\mathrm{Pa}$ \\
\hline $\operatorname{sgn}(u)$ & $\begin{array}{l}\text { signe de } u \text { : } \\
\qquad\left\{\begin{array}{l}\operatorname{sgn}(u)=+1 \text { si } u>0 \\
\operatorname{sgn}(u)=-1 \text { si } u<0\end{array}\right.\end{array}$ & \\
\hline$D_{11}$ & $D_{\prime \prime}=\frac{1}{2}\left((\operatorname{tr} \underline{\underline{D}})^{2}-\operatorname{tr}(\underline{\underline{D}})^{2}\right)$ & \\
\hline
\end{tabular}

car les avalanches en aérosol nécessitent une approche radicalement différente, comme le montre succinctement le paragraphe 2.5 .

Le but de cet article est de présenter un panorama des modèles actuels, que l'on classe selon la loi rhéologique employée. Puis, dans un deuxième temps, on détaille l'écriture des équations du mouvement pour un fluide de Bingham; la simple présentation de ce modèle, appelé AVALDENS au CEMAGREF, illustre les nombreuses difficultés survenant dans la modélisation des avalanches denses. Ensuite, sont présentés les principaux résultats numériques du modèle AVALDENS. Enfin, un dernier chapitre traite de problèmes annexes.

\section{Revue des modèles dynamiques}

Un modèle dynamique consiste en un système d'équations qui représente le mouvement de l'avalanche. La résolution de ce système nécessite la définition d'un certain nombre de paramètres caractérisant la neige en mouvement et des conditions initiales. Pour obtenir le jeu d'équations gouver- 
nant le mouvement, au moins deux approches distinctes sont possibles.

La première méthode consiste à déterminer la loi de comportement de la neige en mouvement, puis à l'injecter dans les équations locales du mouvement (équations de Cauchy) en supposant que la neige est un milieu continu (fluide). Ces équations sont composées d'une équation de conservation de la masse et des équations de conservation de la quantité de mouvement :

$$
\left\{\begin{array}{l}
\frac{\partial \rho}{\partial t}+\operatorname{div}(\rho \vec{u})=0 \\
\rho \frac{\mathrm{d} \vec{u}}{\mathrm{~d} t}=\rho \vec{g}+\operatorname{div} \underline{\underline{T}} .
\end{array}\right.
$$

La seconde méthode intègre sur un volume de contrôle les équations locales sans se soucier de la loi de comportement. En précisant la forme des contraintes sur la frontière du domaine (contraintes à la paroi, pression), on obtient les équations globales du mouvement :

$$
\iiint_{\Omega} \rho \frac{\mathrm{d} \vec{u}}{\mathrm{~d} t} \mathrm{~d} v=\vec{g} \iiint_{\Omega} \rho \mathrm{d} v+\iint_{\partial \Omega} \underline{\underline{T}} \cdot \vec{n} \mathrm{~d} s .
$$

\subsection{Ecriture des équations locales : différentes lois de comportement proposées}

L'écriture des équations locales du mouvement nécessite de définir les relations entre contraintes et déformations au sein du matériau en écoulement : l'objet de ce paragraphe est de décrire les différentes lois de comportement qui ont été proposées pour la neige et les résultats des modèles associés.

\subsubsection{Le modè newtonien}

Le modèle newtonien est la loi rhéologique la plus simple : elle exprime la proportionnalité des contraintes de cisaillement avec la déformation. Tensoriellement. la loi s'écrit pour un fluide incompressible :

$$
\underline{\underline{T}}=-p \underline{\underline{\underline{I}}}+2 \mu \underline{\underline{D}} \text {. }
$$

Quelques auteurs |DENT. 1980|. |DENT. LANG. 1983|. [LANG et Brows. 1980] ont supposé que l'avalanche est un écoulement laminaire d'un fluide incompressible newtonien. Les équations locales du mouvement sont appelées classiquement équations de Navier-Stockes. On adjoint au système dynamique les conditions aux limites pour la surface libre et pour la surface de glissement qui sont elles-mêmes des inconnues du problème. La résolution a été faite numériquement par la méthode des différences finies.

Ces modèles proposés constituent un progrès par rapport aux modèles antérieurs (comme celui de Vollmy) en s'affranchissant d'hypothèses restrictives comme l'assimilation de l'avalanche à son centre de masse ; les comparaisons avec des avalanches réelles ont donné satisfaction à leurs auteurs en ce qui concerne les vitesses atteintes par l'écoulement. Les prédictions de la zone d'arrêt sont médiocres. En effet, il est peu probable que la neige en mouvement soit assimilable à un fluide incompressible newtonien : les écoulements de neige ne sont pas isochores [CARRY. POCHAT, 1978| et le comportement rhéologique est éloigné du comportement newtonien. Ce dernier point a imposé aux auteurs de donner des conditions arbitraires de départ et d'arrêt de l'avalanche. En outre, malgré quelques essais en laboratoires, les auteurs n'ont ni précisé si les écoulements naturels étaient laminaires ou non, ni proposé un critère de transition vers la turbulence. Toutefois, ces premiers modèles furent la porte ouverte à des investigations de plus en plus poussées, qui s'intéressèrent essentiellement à préciser la loi de comportement de la neige en écoulement.

\subsubsection{Le modèle binghamien}

Le modèle de Bingham est une bonne approximation du comportement de beaucoup de fluides naturels [STADLER et Buggisch, 1985], [Buggisch et Stadler, 1986] et il est donc souvent utilisé pour des écoulements géophysiques. Les premiers auteurs à avancer un modèle binghamien furent les américains DENT et LANG en 1983, qui par la suite modifièrent la loi de comportement en donnant un caractère bivisqueux à la contrainte de cisaillement (dans le cas d'un cisaillement simple) [DENT, LANG, 1983].

L'idée essentielle associée à la notion de fluide de Bingham est l'existence d'un seuil de contrainte, c'est-à-dire qu'il est nécessaire d'exercer une contrainte de cisaillement supérieure à une valeur critique $\tau_{\text {c }}$ pour qu'il y ait écoulement : la loi de Bingham est la loi la plus simple qui puisse prendre en compte le caractère viscoplastique d'un matériau. La loi de comportement peut s'écrire [PIAU, 1979]:

$$
\left\{\begin{array}{c}
\underline{\underline{T}}=-p \underline{\underline{l}}+2 \mu \frac{\tau_{\mathrm{c}}}{\sqrt{-D_{\prime \prime}}} \underline{\underline{D}} \text { si } \sqrt{-T_{\| \prime}}>\tau_{,} \\
\underline{\underline{D}=0} \text { si } \sqrt{-T_{\| \prime}}<\tau
\end{array}\right.
$$

où $\sqrt{-T_{\|}}, \sqrt{-D_{\|}}$sont les deuxièmes invariants respectivement du tenseur des contraintes et du tenseur des déformations. On explicite plus simplement cette loi dans le cas d'un écoulement simplement cisaillé et unidirectionnel (fig. I) :

$$
\tau=\tau_{\mathrm{c}}+\mu \frac{\partial u}{\partial y} .
$$

Une des propriétés remarquables d'un fluide de Bingham est l'existence d'une zone non cisaillée, appelée encore plug. où la vitesse est constante [PIAU, 1979]. Les expérimentateurs ont donc tenté de mettre en évidence le plug dans des écoulements de neige en canal; ces expériences ont été menées au moins par deux fois [DENT, LANG, 1983]. [MAENo, Nishimura, 1989], mais les résultats doivent être regardés avec beaucoup de circonspection. Entre autres, il est à noter que :

- les mesures de vitesse ont été faites par procédé optique (caméra à grande vitesse de balayage) et ne peuvent donc concerner que le front de l'écoulement

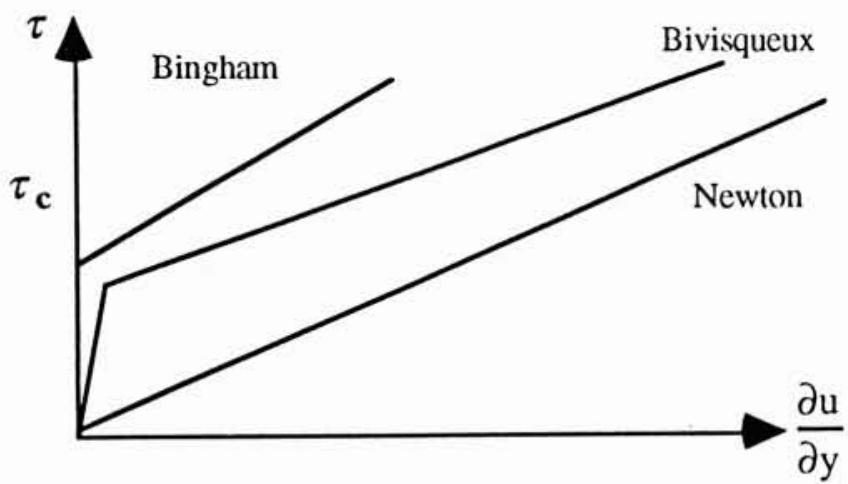

1. Différentes lois de comportement pour un écoulement simplement cisaillé et unidimensionnel. 
- l'écoulement de quelques kilos de neige dans un canal de quelques mètres est qualitativement très différent d'une avalanche réelle et aucun critère de similitude n'a été dégagé, à ce jour, qui puisse justifier les résultats sur modèle réduit.

Par ailleurs, le modèle de Bingham ne permet pas de déterminer l'état de contrainte pour des déformations nulles, aussi DENT et LANG ont préféré lui substituer un modèle bivisqueux dans leur modèle numérique. D'autres auteurs ont mis à profit cette indétermination de l'état de contraintes pour expliciter les conditions de déclenchement et d'arrêt : l'état de contraintes est alors déterminé comme dans le cas statique. Ainsi, MARTINET a esquissé un parallèle entre la contrainte critique $\tau_{c}$ et la contrainte de cisaillement à la rupture, reliée classiquement à la contrainte normale par la loi de Coulomb $\left(\tau_{c}=C+\sigma_{n} \cdot \tan \phi\right)$ [MARTINET, 1992]. Ces analogies sont à considérer avec circonspection, car la neige subit une profonde déstructuration mécanique entre son état initial (stratification du manteau) et sa phase d'écoulement et il est préférable de distinguer la contrainte critique dynamique qui traduit une certaine cohésion du fluide (écoulement en plug) et la contrainte de cisaillement à la rupture d'un bloc de neige soumis à de petites sollicitations.

Nous reviendrons ultérieurement sur le modèle de Bingham puisqu'il est le modèle rhéologique que nous avons choisi.

\subsubsection{Le modèle de Reiner-Rivlin}

De nombreuses théories prennent en compte le caractère visqueux d'un matériau en généralisant le concept de fluide newtonien : les fluides de Reiner-Rivlin en sont un exemple. H. NOREM a proposé plusieurs formulations pour décrire les écoulements de neige et il aboutit à une loi de comportement complexe de la forme [NOREM, 1986, 1989, 1992] :

$$
\underline{\underline{T}}=-p \underline{\underline{I}}+f(\underline{\underline{D}}) \underline{\underline{D}}+g(\underline{\underline{D}}) \underline{\underline{D}}^{2}
$$

où $\underline{\underline{D}}$ est le tenseur des déformations et $f, g$ deux fonctions de ce tenseur. Il simplifie la forme de la loi de comportement pour aboutir à une expression des contraintes en fonction du carré du gradient de vitesse $\dot{\gamma}^{2}$. Un calage de ce modèle avec les expériences sur des suspensions concentrées de SAVAGE et SAYEd a été établi par NOREM [SAVAGE, SAYED, 1984]. Il a comparé les résultats théoriques et les résultats expérimentaux d'après des mesures de H. GÜBLER ou des mesures effectuées à Ryggfonn [NOREM, 1992] : d'après l'auteur, les prédictions du modèle sont correctes. Néanmoins, si cette formulation peut tenir compte de tous les types de neige par l'introduction d'une cohésion (nulle pour un écoulement $\mathrm{sec}$ ), il n'est pas précisé quel type d'avalanches (dense, aérosol) peut être décrit. Par ailleurs, le calage d'un tel modèle par analogie avec les expériences de BAGNOLD ou Savage [Bagnold, 1954], [SAVAGe, SAYEd, 1984] suppose auparavant d'une part que les écoulements de neige soient comparables aux suspensions de billes utilisées par BAGNOLD, et d'autre part que les vitesses de cisaillement soient très importantes pour que le modèle de Bagnold soit applicable, ce qui n'est pas démontré expérimentalement pour les écoulements denses de neige ; de plus, ce modèle ne fournit aucun critère d'arrêt ou de départ. Enfin, cette modélisation sophistiquée nécessite l'introduction de nombreux paramètres (viscosités, coefficients de cisaillement, cohésion, coefficients de frottement...) qui rendent plus complexes le calage et l'utilisation du modèle.

\subsection{Ecriture des équations globales du mouvement}

\subsubsection{Intégration des équations locales}

Si l'on connaît la loi de comportement, on peut obtenir les équations globales du mouvement par intégration des équations locales sur un volume de contrôle, qui est lui-même une inconnue du problème. Pour résoudre le système, il est ensuite nécessaire de préciser la topographie du site, les hauteurs initiales de neige et dans certains cas de donner des conditions arbitraires de départ et d'arrêt de l'avalanche.

Le cas échéant, pour établir les équations du mouvement, on peut utiliser directement la relation (2) sous forme intégrale en précisant les contraintes exercées sur les frontières du domaine. Les modèles de Vællmy et de Hutter \& Savage sont basés sur ce principe.

\subsubsection{Le modèle de Vallmy}

Le modèle le plus ancien et aussi le plus utilisé de nos jours dans l'ingénierie est dû à Vœllmy, qui proposa un modèle basé sur les écoulements stationnaires à surface libre en canal et l'a étendu à des pentes ouvertes (avalanche de versant) ; les hypothèses sont similaires à celles posées en hydraulique fluviale pour des régimes permanents. L'équation du mouvement de l'avalanche est :

$$
M \frac{\mathrm{d} \vec{v}}{\mathrm{~d} t}=M \vec{g}+\vec{F}_{f}+\vec{N}
$$

où la masse de l'avalanche a été assimilée à une masse ponctuelle $M$ située au centre de gravité de l'écoulement supposé en bloc, où $N$ désigne la composante normale de la réaction et où $F$, désigne la force résultante de frottement ; la force de frottement écrite sous la forme :

$$
F_{f}=M(\underbrace{\frac{g v^{2}}{R_{H} \xi}}_{\begin{array}{c}
\text { frottement } \\
\text { turbulent }
\end{array}}+\underbrace{r g \cos \theta}_{\begin{array}{c}
\text { frottement } \\
\text { de Coulomb }
\end{array}})
$$

où $(r, \xi)$ sont des constantes déterminées empiriquement, $R_{H}$ est le rayon hydraulique tel qu'on le définit en hydraulique et $\theta$ désigne l'angle de la pente [VelLmy, 1955]. La hauteur étant supposée constante, il n'y a pas de terme de pression. La vitesse maximale atteinte par l'avalanche est alors :

$$
v=\sqrt{\xi R_{H}(\sin \theta-r \cos \theta)} .
$$

La simplicité de cette équation, le très grand nombre de calages des coefficients de frottement réalisés pour de nombreux sites à travers le monde, les résultats satisfaisants pour l'estimation de la vitesse maximale de l'avalanche et les dimensions de la zone d'arrêt expliquent le succès de ce modèle qui reste le plus utilisé et le plus étudié. De nombreuses améliorations ont été introduites par différentes équipes de chercheurs pour affiner le modèle en introduisant des expressions de frottement différentes... [GÜBLER, 1989], [PERLA et al., 1981], [MARTINELli et LANG, 1980], [EgLit, 1984]. Un opuscule de l'institut suisse EISL présente une méthode didactique et de nombreux exemples pratiques [SALM, BURKARD, GüBler, 1990]. Ces résultats s'appliquent aussi bien aux avalanches denses qu'en aérosol en changeant les paramètres de frottement. 


\subsubsection{Le modèle de Hutter et Savage}

Composées de blocs de neige, les avalanches denses peuvent être assimilées à des écoulements granulaires. La recherche de la loi de comportement des matériaux granulaires fait l'objet de nombreuses études théoriques, comme les travaux de S.B. Savage qui s'est assez largement inspiré de la théorie cinétique des gaz [SAVAGE, 1984]. Parallèlement, il a réalisé de nombreuses expériences sur les avalanches de grains avec la collaboration de HUTter [SAVAGE, HUTTER, 1989, 1991], [HUTTER, 1986, 1989]. Les résultats théoriques ont été obtenus par résolution numérique des équations du mouvement (2) écrites dans un repère curviligne lié à la pente, en supposant que le nombre d'aspect $\varepsilon$ (hauteur sur longueur d'écoulement) et le coefficient de courbure $\lambda$ sont faibles. En ce qui concerne les contraintes exercées sur les frontières du domaine (une tranche de fluide d'épaisseur $\mathrm{d} \xi$ ), le frottement à la paroi est supposé proportionnel au carré de la vitesse moyenne; la pression exercée est localement proportionnelle à la contrainte normale par l'intermédiaire du coefficient $\kappa_{\text {actpass }}$ défini sur le cercle de Mohr-Coulomb à partir de $\delta$ l'angle de frottement à la paroi (voir fig. 2). Les notations sont explicitées sur la figure 4. Finalement, on aboutit à :

$$
\left\{\begin{array}{l}
\frac{\partial h}{\partial t}+\frac{\partial h \bar{u}}{\partial \xi}=0 \\
\left(\frac{\partial \bar{u}}{\partial t}+\bar{u} \frac{\partial \bar{u}}{\partial \xi}\right)=g \sin \theta-\operatorname{sgn}(u) \times \\
\times\left(g \cos \theta+\lambda C u^{2}\right) \tan \delta-\varepsilon \kappa_{\text {actpass }} \cos \theta g \frac{\partial h}{\partial \xi}
\end{array}\right.
$$

où $C$ est la courbure de la pente (fig. 2). Des expériences ont été réalisées sur un canal incliné et rugueux en laissant s'écouler une masse de billes en plastique, mais à notre connaissance aucune comparaison avec des avalanches réelles n'a été effectuée. L'avantage de cette approche est qu'elle est applicable en théorie à de nombreux autres écoulements géophysiques (laves torrentielles, éboulements) ; de plus sa concision (il suffit de connaître les angles de frottement interne et à la paroi pour déterminer tous les paramètres des

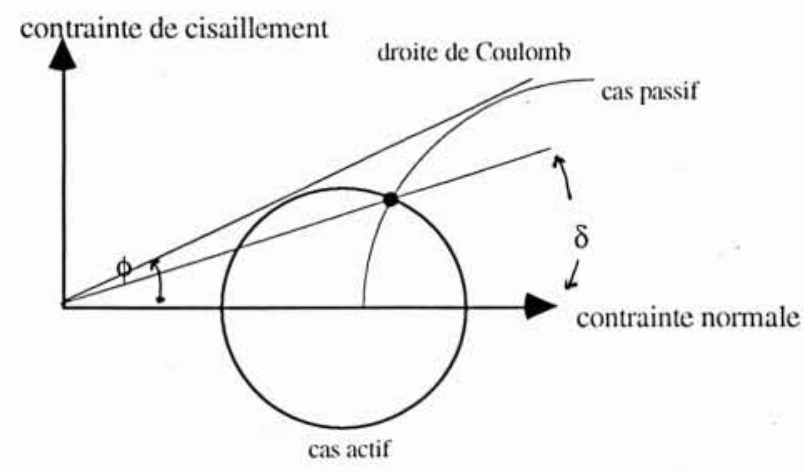

2. Diagramme de Mohr-Coulomb selon Savage, qui permet de déterminer l'état de contrainte en connaissant l'angle de frottement interne $f$, l'angle de frottement de la paroi $d$ et la condition de contraintes (actif ou passif selon que la rupture se fasse en butée ou en poussée). équations du mouvement) en fait un modèle pratique. II convient toutefois de noter que l'introduction du coefficient de pression $\kappa_{\text {actpass }}$ suppose que la répartition des contraintes est totalement influencée par le frottement pariétal, ce qui n'est pas expérimentalement une évidence.

\subsection{D'autres approches}

Bien d'autres approches ont été tentées pour prévoir les risques d'avalanches et localiser les zones d'extension. Pour mémoire, on peut citer diverses méthodes statistiques mises en œuvre dans plusieurs pays [MAC CLUNG, LIED, 1987], [LIED, TOPPE, 1989], [BUSER, 1989] ou des systèmes à base de connaissances qui développent des techniques d'intelligence artificielle pour reproduire et quantifier le jugement d'un expert [Bolognesi, 1991], [Buisson, Charlier, 1989]. Ces modèles sont soit des méthodes de prévision du risque d'avalanche, soit des outils performants capables de tenir compte des caractéristiques d'un site (végétation, zone d'accumulation, histoire météorologique...), d'intégrer le raisonnement d'un expert et d'adjoindre un code de calcul classique.

\subsection{Conclusions}

Le rapide développement des moyens informatiques et l'amélioration des techniques de résolution numérique ont permis d'élaborer des modèles comportant des lois de comportement de plus en plus sophistiquées. Néanmoins, la validation tant des lois rhéologiques que des modèles dynamiques est trop largement insuffisante. En effet, il faut savoir que :

- les essais in situ sont rares, non-reproductibles et il est difficile de mesurer d'une manière fiable et complète leurs caractéristiques cinématiques et dynamiques. La plupart des données disponibles concernent la vitesse du front ou les dimensions de la zone de dépôt,

- les essais en laboratoire doivent être considérés avec prudence tant que des critères de similitude entre écoulements naturel et reproduit ne seront clairement explicités : il n'est pas évident que le glissement d'un paquet de neige (ou d'un autre matériau) soit représentatif d'une avalanche réelle. Par ailleurs, la neige est un matériau particulièrement pénible à étudier en laboratoire lors d'essais au triaxial ou en rhéomètre car de nombreux phénomènes viennent parasiter les mesures (fusion, frittage de contact, métamorphose...) [MONTMOLLin, 1978].

Ces quelques faits expliquent la profusion de modèles basés sur des lois rhéologiques différentes, car aucune expérience sérieuse, à l'heure actuelle, $\mathrm{n}$ 'a mis en évidence la loi de comportement de la neige : les modèles proposés ne sont que des conjectures.

\subsection{Le cas des avalanches en aérosol}

Ce dernier paragraphe traite rapidement le cas des avalanches en aérosol: ainsi, on espère montrer la grande différence entre la dynamique des écoulements denses et en aérosol, distinction qui n'est malheureusement que peu souvent ou mal opérée, sans doute à cause de la rareté de grandes avalanches en aérosol dans nos pays au climat tempéré. Par ailleurs, il convient d'éviter un amalgame trop souvent commis entre avalanche en aérosol et avalanche de neige poudreuse: des exemples historiques montrent que des 
accumulations de neige lourde peuvent aussi créer des aérosols (avalanche du 16 mai 1983, Chamonix, voir dans [ANCEY, 1992]).

Enfin, il nous paraît actuellement illusoire de traiter simultanément écoulements denses et en aérosol tant leur mécanique diffère. Pour s'en convaincre, nous reprenons une analyse de BÉGHIN et HOPFINGER [BÉGHIN, 1979], [BÉGHIN, Hopfinger, BritTer, 1981], [BÉGhin, Olagne, 1991] (fig. 3).

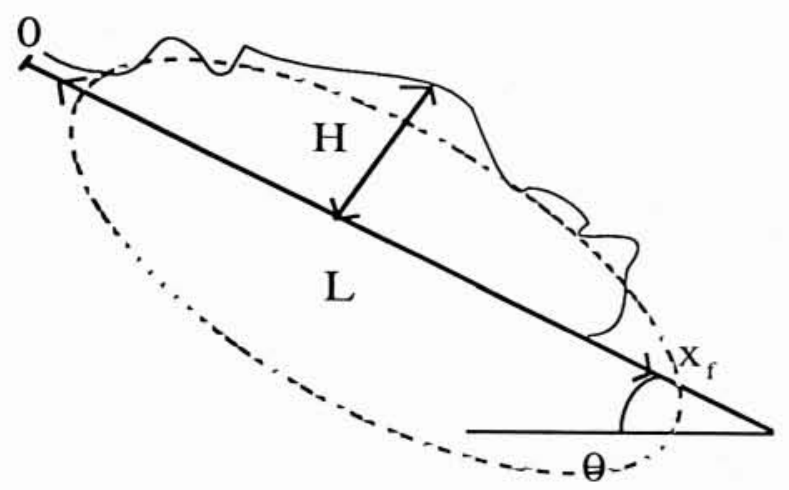

3. Un écoulement en aérosol est représenté géométriquement par une demi-ellipse de grand axe $\boldsymbol{L}$ et de petit axe $\boldsymbol{H}$.

Les résultats théoriques ont été confrontés avec les résultats expérimentaux qui consistent à étudier en canal noyé l'écoulement d'un fluide lourd et miscible. Si peu de comparaisons ont pu être effectuées avec des avalanches réelles, les résultats donnent une idée de la structure turbulente des bouffées de neige. Pour simplifier le problème, on formule les hypothèses suivantes:

- l'écoulement est turbulent

- la variation de la masse volumique entre le nuage $(\bar{\rho})$ et l'air $\left(\rho_{a}\right)$ est négligeable (hypothèse de Boussinesq), la masse de neige est constante dans l'aérosol (pas de sédimentation et pas de reprise)

- la poussée d'Archimède est invariante, les frottements sont négligés

- la forme de l'avalanche (approximée par une demiellipse de grand axe $L$ et de petit axe $H$ ) se conserve durant le mouvement, donc $k=H / L$ est une constante.

Dès lors, en assimilant l'avalanche à son centre d'inertie et en projetant selon $x$, l'équation de quantité de mouvement s'écrit :

$$
\frac{\mathrm{d} \rho_{a}\left(1+k_{v}\right) S_{1} H L U}{\mathrm{~d} t}=B \sin \theta
$$

où $B$ est la poussée d'Archimède $B=g\left(\bar{\rho}-\rho_{a}\right) H L S_{1}, \theta$ l'angle de pente, $S_{1}$ est un facteur de forme défini comme étant la surface de la section transversale divisée par $H L$, $k_{v}$ un coefficient de masse équivalente, $U$ est la vitesse. De même, l'équation de conservation de la masse s'écrit en tenant compte du fait que la variation de la masse totale de l'aérosol est due à l'incorporation d'air avec une vitesse d'entraînement proportionnelle à $U$ :

$$
\frac{\mathrm{d} S_{1} H L}{\mathrm{~d} t}=S_{2} \sqrt{H L} \alpha(\theta) U
$$

où $S_{2}$ est l'aire de la surface délimité par l'ellipsoïde divisée par $(H L)^{1 / 2}$. Un choix judicieux serait par exemple :

$$
S_{1}=\frac{\pi}{4}, \quad S_{2}=\frac{\pi}{2^{\frac{2}{3}}} \frac{\sqrt{1+4 k^{2}}}{\sqrt{k}} .
$$

L'intégration de l'équation de la masse donne :

$$
\begin{aligned}
& H=\frac{1}{2} \frac{S_{2}}{S_{1}} \sqrt{k} \alpha x \\
& L=\frac{1}{2} \frac{S_{2}}{S_{1}} \frac{1}{\sqrt{k}} \alpha x .
\end{aligned}
$$

On reporte ces valeurs dans l'équation du mouvement, que l'on intègre pour obtenir :

$$
U=\sqrt{U_{0}^{2}\left(\frac{x_{0}}{x}\right)^{4}+\frac{2 C}{3}\left(1-\left(\frac{x_{0}}{x}\right)^{3}\right) \frac{1}{x}}
$$

où l'indice zéro réfère aux conditions initiales et où $C=\frac{4 S_{1} B}{\rho_{a}\left(1+k_{v}\right) \alpha^{2} S_{2}^{2}} \sin \theta$. Cette expression de la vitesse permet de montrer qu'il existe tout d'abord une phase d'accélération, puis une phase de décélération (à condition que $U_{0}<\sqrt{2 C / 3 x_{0}}$ ). Asymptotiquement (avec $U_{0}=0$ ), la vitesse est de la forme :

$$
U=\sqrt{\frac{2 C}{3} \frac{1}{x}} .
$$

Les auteurs certifient qu'un tel modèle très simple est capable de représenter dans ses grandes lignes l'écoulement en aérosol, dont les caractéristiques dynamiques (vitesse et hauteur d'écoulement par exemple) diffèrent des caractéristiques des écoulements denses. Par conséquent les effets des deux types d'écoulements (pression d'impact, zone d'arrêt, onde de choc due au mouvement d'air...) ne sont pas semblables [HOPFINGER, 1983].

\section{Le modèle AVALDENS}

L'objectif du programme entrepris par le CEMAGREF depuis bientôt une dizaine d'années est la réalisation, entre autres, d'un modèle numérique bidimensionnel simulant les avalanches denses. Cet article présente le point des réalisations actuelles.

\subsection{Hypothèses physiques du modèle $1 D$}

Nous ne reviendrons pas sur les définitions adoptées concernant les écoulements denses, dont le premier paragraphe s'est fait l'écho. On retiendra que l'on assimile la neige à un fluide incompressible de Bingham. C'est une hypothèse de travail, qui présente de nombreux avantages comme la prise en compte d'un seuil de contrainte (qui permet une mise en mouvement de la neige ou son arrêt à partir d'une contrainte 


\section{MODÉLISATION DES AVALANCHES DENSES}

critique), même si la réalité physique est nettement plus complexe. La formulation mathématique qui suit ne perd pas de sa généralité si l'on choisit une loi de comportement différente. Par ailleurs, une série de tests numériques sur AVALDENS a été lancée pour délimiter le domaine de validité de l'hypothèse d'incompressibilité comme on le verra par la suite.

En outre, nous supposerons que l'avalanche est entièrement déterminée par son mode d'écoulement à l'entrée de la zone d'écoulement et n'est pas influencée par le type de départ (départ ponctuel ou en "plaque ") ou la qualité de la neige, c'est-à-dire que l'on suppose que le brassage de la neige durant son écoulement dans la zone d'accumulation est tel que la neige a perdu la mémoire de son état (mécanique) d'origine.

Les équations du mouvement unidirectionnel de l'avalanche sont obtenues par intégration des équations de conservation de la quantité de mouvement et de la masse. Ce travail est inspiré de calculs analogues effectués en Hydraulique Fluviale (équations de Saint Venant). Ainsi, on suppose une répartition hydrostatique de la pression, ce qui peut se légitimer en considérant que les variations de la surface libre de l'écoulement sont faibles (hormis au niveau du front) et que la hauteur d'écoulement est petite devant sa longueur. Enfin, on suppose que l'écoulement est simplement cisaillé et que les variations de pente ne sont pas brutales.

\subsection{Equations du mouvement}

\subsubsection{Expression dans le repère lié au sol}

On va écrire les équations de conservation de la masse et de la quantité de mouvement en coordonnées curvilignes $(\xi, \eta)$ dans le repère lié à la ligne de pente $C_{f}$ représentée par une fonction de la forme $y=f(x)(f i g .4)$.

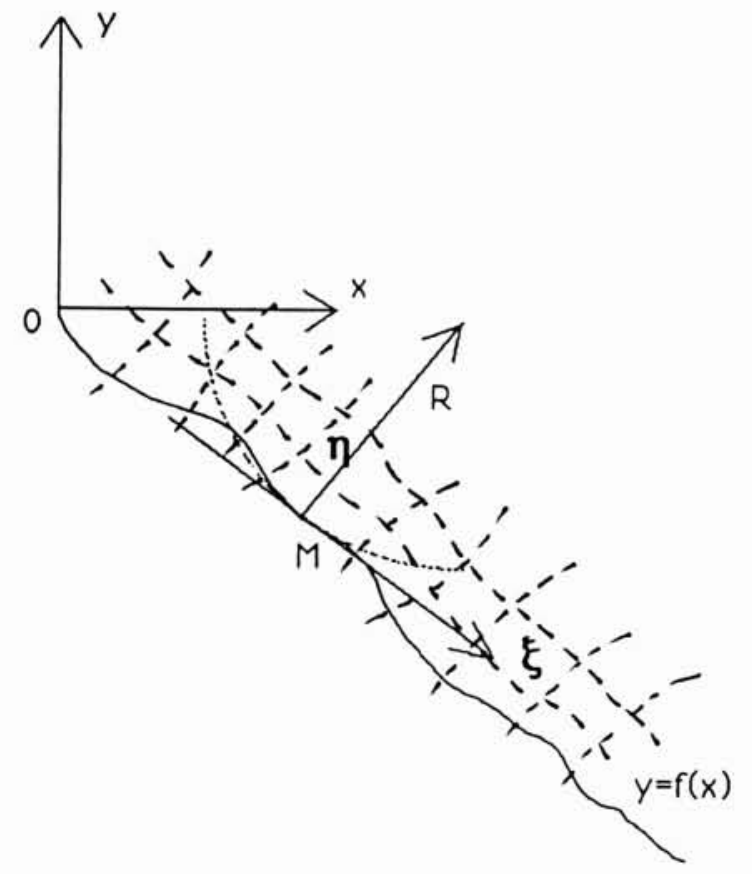

4. Coordonnées curvilignes adaptées à la description d'un écoulement sur une configuration de terrain unidirectionnelle.
Dans ce repère, $\xi$ représente l'abscisse curviligne et $\eta$ l'ordonnée mesurée selon la normale orientée à $C_{f}$; les composantes de la vitesse sont $(u, v)$ et on note $R$ le rayon de courbure et $C$ la courbure :

$$
C=\frac{1}{R}=\frac{f^{\prime \prime}(x)}{\sqrt{1+f^{\prime 2}(x)}} .
$$

Le tenseur métrique s’écrit :

$$
\underline{\underline{g}}=\left[\begin{array}{cc}
(1-C \eta) & 0 \\
0 & 1
\end{array}\right] \text {. }
$$

L'équation de continuité est :

$$
\frac{\partial u}{\partial \xi}+\frac{\partial(1-C \eta) v}{\partial \eta}=0 .
$$

Les équations de quantité de mouvement projetées selon

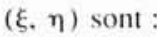

$$
\left\{\begin{array}{r}
\rho\left(\frac{\partial u}{\partial t}+\frac{u}{1-\eta C} \frac{\partial u}{\partial \xi}+v \frac{\partial u}{\partial \eta}-\frac{C u v}{1-\eta C}\right)=\rho g \sin \theta+ \\
+\frac{1}{1-\eta C}\left(\frac{\partial T_{\xi \xi}}{\partial \xi}+\frac{\partial(1-C \eta) T_{\xi \eta}}{\partial \eta}\right)+\frac{C T_{\xi \eta}}{1-C \eta} \\
\rho\left(\frac{\partial v}{\partial t}+\frac{u}{1-\eta C} \frac{\partial v}{\partial \xi}+v \frac{\partial v}{\partial \eta}+\frac{C u^{2}}{1-\eta C}\right)=-\rho g \cos \theta+ \\
+\frac{1}{1-\eta C}\left(\frac{\partial T_{\eta \xi}}{\partial \xi}+(1-C \eta) \frac{\partial T_{\eta \eta}}{\partial \eta}+C\left(T_{\eta \eta}-T_{\eta \xi}\right)\right) . \\
(21 a, b)
\end{array}\right.
$$

\subsubsection{Simplification, intégration des équations}

On va simplifier le problème précédent en supposant que le rayon de courbure est toujours grand et positif. ce qui impose à la ligne de pente d'être régulière sans variation brutale. Dès lors, on peut négliger $C$ devant 1 . On retrouve alors les équations du mouvement établies pour un écoulement infini le long d'un plan incliné d'angle $\theta$ avec l'horizontale :

$\left\{\begin{array}{c}\frac{\partial u}{\partial \xi}+\frac{\partial v}{\partial \eta}=0 \\ \rho\left(\frac{\partial u}{\partial t}+u \frac{\partial u}{\partial \xi}+v \frac{\partial u}{\partial \eta}\right)= \\ \rho\left(\frac{\partial v}{\partial t}+u \frac{\partial v}{\partial \xi}+v \frac{\partial v}{\partial \eta}\right)=-\rho g \cos \theta+\frac{\partial T_{\eta \xi}}{\partial \xi}+\frac{\partial T_{\xi \eta}}{\partial \eta} \\ \partial \eta \\ (22 a, b, c)\end{array}\right.$

Par commodité, on remplace $(\xi, \eta)$ par les coordonnées $(x, y)$. On va intégrer ce système selon la normale $(M y)$ en tenant compte des conditions aux limites de la surface libre : — imperméabilité de la surface libre :

$$
v(h)=\frac{\mathrm{d} h}{\mathrm{~d} t}=\frac{\partial h}{\partial t}+u \frac{\partial h}{\partial y}
$$

- aucune contrainte imposée en surface :

$$
T \cdot \bar{n}=0 .
$$


On aboutit alors au système suivant en notant

$$
\begin{gathered}
\langle g\rangle=\int_{0}^{h} g(y) \text { d } y \text { et } \bar{g}=\frac{1}{h}\langle g\rangle: \\
\frac{\partial h}{\partial t}+\frac{\partial\langle u\rangle}{\partial x}=0 \\
\left\{\begin{array}{r}
\rho\left(\frac{\partial\langle u\rangle}{\partial t}+\frac{\partial\left\langle u^{2}\right\rangle}{\partial x}\right)=\rho g h \sin \theta+\frac{\partial\left\langle T_{x x}\right\rangle}{\partial x}-T_{1} \\
\rho\left(\frac{\partial\langle v\rangle}{\partial t}+\frac{\partial\left\langle v^{2}\right\rangle}{\partial x}\right)=-\rho g h \cos \theta+\frac{\partial\left\langle T_{x y}\right\rangle}{\partial x}-T_{2} \\
(25 a, b, c)
\end{array}\right.
\end{gathered}
$$

$\operatorname{avec}\left(T_{1}, T_{2}\right)=\left(T_{x y}(0), T_{y y}(0)\right)$.

\subsubsection{Equations du modèle ID}

Dans le cas d'un fluide de Bingham, en supposant la répartition de pression hydrostatique (parallèle au fond), les équations du mouvement ID se réduisent au système suivant :

$$
\left\{\begin{array}{c}
\frac{\partial h}{\partial t}+\frac{\partial h \bar{u}}{\partial x}=0 \\
\rho\left(\frac{\partial h \bar{u}}{\partial t}+\frac{\partial\left(h \overline{u^{2}}+\frac{1}{2} g h^{2}\right)}{\partial x}\right)= \\
=\rho g h \sin \theta-\tau_{c}-\mu \frac{\partial u}{\partial y}(0) .
\end{array}\right.
$$

Afin d'obtenir une équation où n'intervient que la vitesse moyenne de l'écoulement, on va essayer de trouver une relation entre $\bar{u}, \overline{u^{2}}$ et la contrainte à la paroi. Si l'écoulement est stationnaire on montre facilement que le profil de vitesse est, en notant $y_{0}$ la hauteur de la zone nón cisaillée :

$$
\begin{aligned}
& \text { si } y_{0} \leqslant y \leqslant h \text { alors : } \\
& u(y)=u^{*}=\frac{\rho g \sin \theta}{2 \mu} \quad y_{0}^{2}=\frac{\tau_{c}}{2 h_{c} \mu} y_{0}^{2} \\
& \text { si } 0 \leqslant y \leqslant y_{0} \text { alors : } \\
& \begin{aligned}
& u(y)=\frac{\rho g \sin \theta}{\mu}\left(h y-\frac{y^{2}}{2}\right)-\frac{\tau_{c}}{\mu} y= \\
&=u^{*}\left(2 \frac{y}{y_{0}}-\frac{y^{2}}{y_{0}^{2}}\right)=u^{*} \varphi\left(\frac{y}{y_{0}}\right) .
\end{aligned}
\end{aligned}
$$

Le profil de vitesse dans la zone non cisaillée présente la remarquable propriété d'être auto-similaire.

On en déduit que :

$$
\frac{\bar{u}}{u^{*}}=\frac{2}{3}+\frac{h_{c}}{3 h}
$$

où $h_{c}$ est la hauteur critique, c'est-à-dire la hauteur minimale nécessaire pour qu'il y ait mouvement : $h_{c}=\tau_{c} /(\rho g \sin \theta)$. La contrainte à la paroi vaut :

$$
\tau=\rho g h \sin \theta=\tau_{i}+\mu \frac{\partial u}{\partial y}(0)=\tau_{c}+2 \mu \frac{u^{*}}{y_{0}}
$$

tandis que la relation liant les moyennes de la vitesse et du carré de la vitesse est :

$$
\frac{\overline{u^{2}}}{\bar{u}^{2}}=\frac{9}{5} \frac{6-\eta}{(2+\eta)^{2}}
$$

où $\eta=h_{f} / h$. Ce rapport est toujours compris entre 1 et 2,7 .
On supposera toutefois en vue de simplifier le problème dans ce qui suit que ce rapport vaut 1 (fig. 5).

Quand l'écoulement n'est pas stationnaire, on supposera que les relations précédentes restent vérifiées. L'équation (26b) s'écrit alors :

$\rho\left(\frac{\partial h \bar{u}}{\partial t}+\frac{\partial h \overline{u^{2}}+\frac{1}{2} g h^{2}}{\partial x}\right)=\rho g h \sin \theta-\tau_{c}-L(h, \theta) \bar{u}$

où l'on a noté :

$$
L(h, \theta)=2 \mu \frac{1}{1-\frac{y_{0}}{3 h}} \frac{1}{y_{0}}=\frac{2 \mu}{\left(h-h_{c}\right)\left(\frac{2}{3}+\frac{h_{c}}{3 h}\right)} .
$$

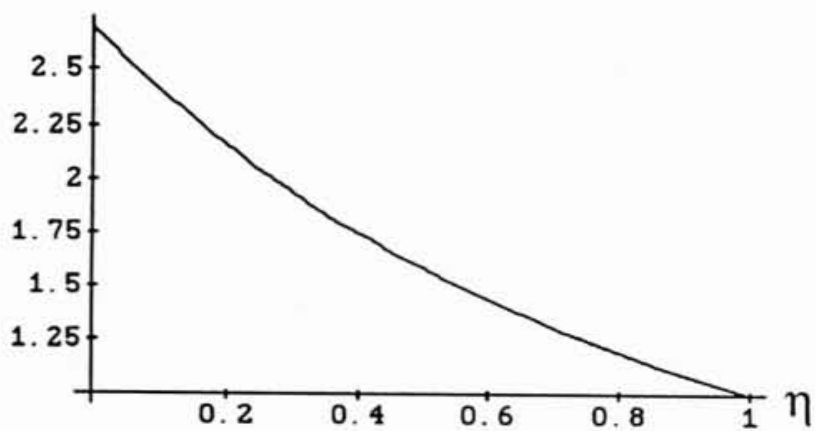

5. Variation de $\overline{u^{2}} / \bar{u}^{2}$ en fonction de $\eta$.

\subsubsection{Ecoulements laminaires et turbulents}

Classiquement, les auteurs s'accordent à dire que les écoulements de neige sont turbulents sans pourtant apporter d'argument à leur assertion. Pour notre part, les observations sur les avalanches déclenchées au-dessus du col du Lautaret nous amènent à croire que des écoulements laminaires denses existent (les vitesses à la surface de l'avalanche ont été évaluées entre 6 et $10 \mathrm{~m} / \mathrm{s}$, la surface libre est régulière et peu mouvementée) [MARCO, 1993]. Nous allons essayer ici de donner quelques éléments de réponse. En premier lieu, il convient de prendre garde au fait que les mesures de viscosité et de densité de la neige ont été le plus souvent réalisées sur de petits échantillons de neige ; il reste à voir si ces valeurs sont représentatives des écoulements denses qui comportent des boules de neige noyées ou non dans une matrice de neige. Il serait plus adéquat de parler d'une viscosité et d'une masse volumique équivalentes.

De même qu'en hydrodynamique des fluides newtoniens, où il est d'usage d'exprimer la contrainte à la paroi sous la forme :

$$
\tau_{p}=f \rho \frac{u^{2}}{2}
$$

où $f$ est un coefficient de frottement (qui ne dépend que du nombre de Reynolds Re et de la rugosité $\xi$ de la conduite), on écrira le frottement sous cette forme. Dans le cas de fluide de 
Bingham $f$ est en plus une fonction du nombre de Hedstrom (He) [Midoux, 1985]. En régime laminaire et dans notre configuration plane, on peut écrire que :

$$
\tau_{p}=\tau_{c}+L \bar{u}=\tau_{c}+f_{n} \rho \frac{\bar{u}^{2}}{2}
$$

avec :

$$
f_{n}(\operatorname{Re}, \eta)=\frac{6}{\operatorname{Re}} \frac{1}{(2+\eta)(1-\eta)} \quad \text { où } \quad \eta=\frac{h_{c}}{h} .
$$

L'équation (30a) peut s'écrire alors :

$$
\begin{aligned}
\tau_{p}=\left(2 \frac{\mathrm{He}}{\operatorname{Re}^{2}}+\frac{6}{\operatorname{Re}} \frac{1}{(1-\eta)(2+\eta)}\right) \times \\
\quad \times \rho \frac{\bar{u}^{2}}{2}=f(\operatorname{He}, \operatorname{Re}, \eta) \rho \frac{\bar{u}^{2}}{2} .
\end{aligned}
$$

L'expression (37) donne le frottement à la paroi pour un écoulement laminaire en fonction des trois nombres adimensionnels $\mathrm{He}, \mathrm{Re}, \eta$. L'expression en régime turbulent fait encore défaut. En ce qui concerne les écoulements de fluides binghamiens turbulents, plusieurs expériences en conduites ont été réalisées [HANKS, DaDIA, 1971]. [LUMLeY, 1976]. TROWBRIDGE fait une synthèse de ces approches et propose un critère de transition [TRowBridge, 1987]. Les fluides concernés étaient essentiellement des suspensions à faible concentration dans de l'eau, donc des fluides de Bingham avec une faible valeur de la contrainte critique (de l'ordre du Pascal), contrairement aux écoulements de neige (contrainte critique de plusieurs centaines de Pa). Nous conserverons la notation précédente (35) ou (37) qui a l'avantage d'être générale et n'oblige pas à faire d'hypothèse sur la nature de l'écoulement, même si l'expression n'est pas connue dans le cas d'un écoulement turbulent. Nous avons fait de nombreux essais numériques en considérant des écoulements turbulents ou laminaires, qui donnent sensiblement le même résultat après un calage adéquat [ANCEY, 1992]. Un progrès décisif sera réellement accompli quand un critère de transition vers la turbulence aura été proposé pour des fluides de Bingham possédant un seuil de contrainte important.

\subsubsection{Stabilité des écoulements}

En se basant sur une analyse linéaire de la stabilité, TROWBRIDGE propose le critère suivant d'instabilité, qui conditionne l'apparition de trains d'ondes (roll waves) de faibles amplitudes perturbant la surface libre d'un écoulement représenté par l'équation $(26 a, b)$ :

$$
\left(\tau_{P}-H \frac{\partial \tau_{P}}{\partial h}\right)\left(\frac{\partial \tau_{P}}{\partial u}\right)^{-1}>\sqrt{g h \cos \theta}
$$

les calculs montrant alors que l'écoulement devient instable si le nombre de Froude excède la valeur suivante:

$$
\operatorname{Fr}>\frac{3-\eta-\eta^{2}}{2+\eta^{2}}
$$

On peut en conclure que dès que le nombre de Froude devient supérieur à 1,5 l'écoulement devient instable (fig. 6).

\subsubsection{Ecoulement en canal}

L'équation (32) du mouvement est valable pour un écoulement plan et peut donc servir pour des avalanches de versant. Pour des avalanches de couloir, il faut tenir compte des effets

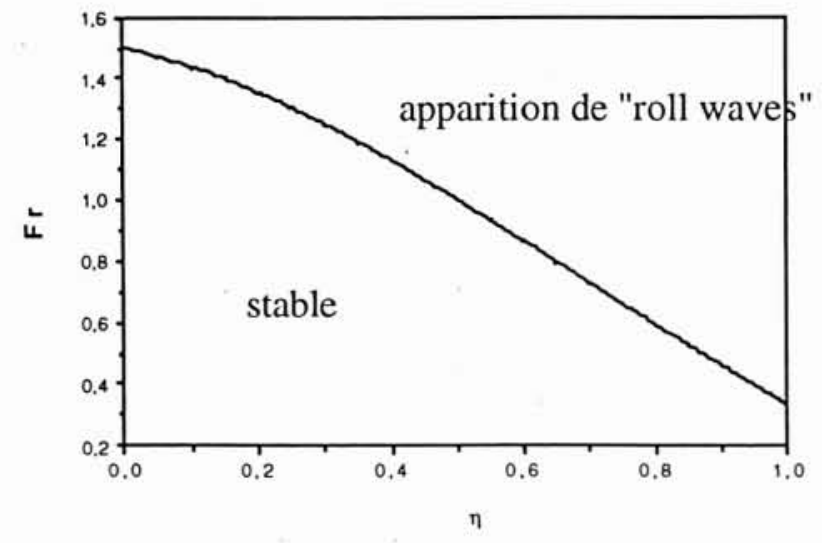

6. Condition d'apparition des « roll waves " en fonction du nombre de Froude et de $\eta$.

de bord; les hypothèses physiques sont identiques. Pour un angle de pente faible et un seuil de contrainte nul, on retombe sur les équations de Saint-Venant. Un bilan des forces sur un volume infinitésimal amène à écrire que :

$$
\left\{\begin{array}{c}
\frac{\partial S}{\partial t}+\frac{\partial(S u)}{\partial x}=0 \Leftrightarrow \frac{\partial S}{\partial t}+\frac{\partial Q}{\partial x}=0 \\
\frac{\partial \rho Q}{\partial t}+\frac{\partial(\rho Q u)}{\partial x}=\rho S g \sin \theta-\left(\rho f g u^{2}+\tau_{c}\right) x-\rho g S \frac{\partial h}{\partial x}
\end{array}\right.
$$

où $Q$ est le débit, $S$ la section mouillée, $x$ le périmètre mouillé. Le système $(40)$ peut se mettre sous la forme:

$$
\frac{\partial \vec{U}}{\partial t}+\frac{\partial \vec{F}(\vec{U}, x)}{\partial x}=G(\vec{U}, x)
$$

où $F$ et $G$ sont deux fonctionnelles. On peut montrer que c'est un système différentiel hyperbolique non linéaire [VILA, 1985].

\subsection{Résolution numérique}

Les équations (41) forment un système non linéaire hyperbolique. LAX a montré qu'une discontinuité de la solution prenait naissance au bout d'un temps fini, il ne peut donc y avoir de solution régulière au problème, ce qui entre en contradiction avec les hypothèses formulées concernant la continuité de la surface libre [VILA, 1985]. On est donc obligé d'adjoindre une équation supplémentaire traduisant la discontinuité de la solution. La solution (au sens des distributions) doit être compatible avec cette nouvelle équation dite équation du ressaut hydraulique.

La résolution numérique est rendue délicate tant du point de vue de son écriture que de sa stabilité. C'est pourquoi on préfère substituer aux équations $(40 a, b)$ le système conservatif sous sa forme faible (41) qui est compatible avec l'équation du ressaut [G. MARTINET, 1992]. Afin de simplifier l'exposé de la méthode, on va dans un premier temps supposer que le terme $G$ est nul : c'est le problème de Cauchy que l'on va transformer en un problème de Riemann. 
On appelle problème de Riemann un problème de Cauchy avec une condition initiale particulière :

$$
\left\{\begin{array}{c}
\frac{\partial U}{\partial t}+\frac{\partial f(U)}{\partial x}=0 \\
\text { en } x=0\left\{\begin{array}{l}
x<0 \Rightarrow U(x, 0)=U g \\
x>0 \Rightarrow U(x, 0)=U d .
\end{array}\right.
\end{array}\right.
$$

Ce problème permet de traiter les discontinuités. On introduit la notion de solutions faibles afin de s'occuper d'une discontinuité comme un point courant. La difficulté essentielle est ensuite de sélectionner la bonne solution, car malheureusement il n'y a pas unicité de la solution. On définit alors le concept d'entropie qui exprime une dissipation de l'énergie due aux chocs et permet de choisir la bonne solution.

Le schéma de Godunov cherche une solution dans l'espace des fonctions constantes sur chaque cellule du maillage : la résolution se ramène alors à une juxtaposition de problèmes de Riemann posés pour chaque interface des mailles. On adjoint une condition sur le pas de temps $\mathrm{d} t$ qui ne peut pas être pris trop grand sinon les ondes issues des interfaces d'une même maille peuvent interagir : c'est la condition dite de COURANT-FRIEDRICH-LÉVY (CFL).

Le nœud de la difficulté est ensuite de trouver une solution du problème de Riemann au temps $t+\mathrm{d} t$ qui appartienne à notre ensemble des fonctions constantes par maille. Godunov a choisi la valeur moyenne de cette fonction sur chaque maille (on parle de projection de la solution sur chaque maille) ; malheureusement, une telle formulation n'est pas numériquement applicable (problème de consistance). On construit alors une solution approchée du problème de Riemann [VILA, 1985], [VILA et al., 1992]. Ces schémas sont appelés «solveurs », et le plus simple est le solveur de $\mathrm{R}$, qui consiste à remplacer l'équation non linéaire

$$
\frac{\partial U}{\partial t}+\frac{\partial f(U)}{\partial x}=0
$$

par une équation linéaire :

$$
\frac{\partial U}{\partial t}+c \frac{\partial U}{\partial x}=0 .
$$

\subsection{Résultats numériques}

On a réalisé plusieurs simulations numériques d'avalanches sur des profils concaves et on reporte ici les principaux résultats.

\subsubsection{Influence de la masse volumique}

Les équations du mouvement ont été établies en supposant que la masse volumique était constante, or les mesures sur le terrain mettent en doute une telle hypothèse [CARRY, POCHAT, 1978]. Nous avons donc considéré une variation fictive de la masse volumique au cours du temps pour voir son influence sur l'écoulement. A cet effet, on a introduit la variation de masse volumique sous la forme d'une fonction dépendant du temps et d'un paramètre de forme $\alpha$, qui permet de faire varier l'allure de la courbe :

$$
\rho=f(t, \alpha)=\rho_{i}+\beta t^{\alpha}\left(\frac{8}{\alpha}-\frac{6}{\alpha+1} t+\frac{t^{2}}{\alpha+2}\right)
$$

où $\beta$ est un coefficient de compactage choisi de telle sorte que :

$$
\rho(t=0)=\rho_{i} \text { et } \rho\left(t=t_{\max }\right)=\rho_{f} .
$$

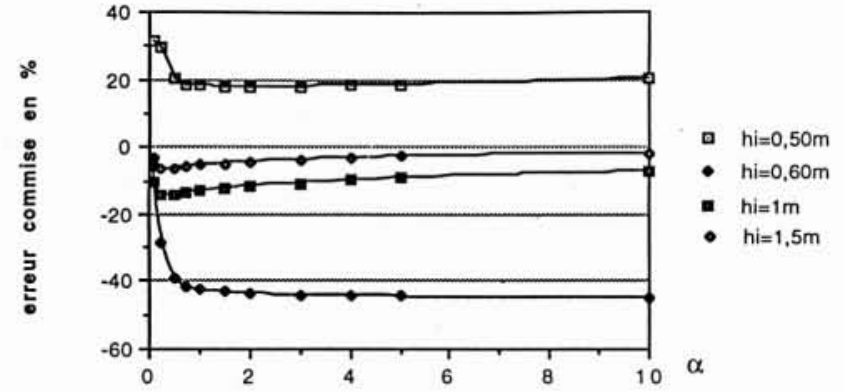

7. Erreur commise sur la vitesse maximale atteinte par l'avalanche pour différentes hauteurs du manteau neigeux en fonction du paramètre de forme $\alpha$.

On a reporté sur la figure 7 ci-dessus la valeur de l'erreur commise entre les vitesses maximales atteintes par l'avalanche à densité constante et à densité variable caractérisée par $\alpha$ pour différentes hauteurs du manteau neigeux. On remarque que la masse volumique n'influe que faiblement sur la vitesse pour de grosses avalanches mais est déterminante si les hauteurs initiales du manteau neigeux sont faibles.

\subsubsection{Influence de la contrainte critique}

Sur la figure 8 sont reportées les variations de la vitesse maximale en fonction de la contrainte critique pour différentes hauteurs de déclenchement. Les courbes ont un comportement semblable : dans un premier temps, il y a une variation quasi linéaire (le coefficient de corrélation est toujours supérieur à 0,9 ), puịs un minimum de la vitesse qui est suivi d'une remontée sensible de la vitesse (ce qui reste peu expliqué), enfin les courbes s'effondrent au-delà d'une contrainte limite qui dépend de la hauteur initiale. La vitesse est le seul paramètre cinématique qui varie de manière conséquente avec la contrainte critique ; les autres paramètres (hauteur d'écoulement, dimensions de la zone d'arrêt) y sont moins sensibles.

Les auteurs qui ont proposé un modèle Binghamien, ont donné des valeurs de contraintes critiques comprises entre 500 et $1500 \mathrm{~Pa}$ [MARTINET, 1992], mais il semble que rien n'empêche d'avoir des contraintes plus élevées. La valeur de $\tau_{c}$ influence la forme de l'écoulement : plus la contrainte est grande, plus l'écoulement devient pâteux et a tendance à se faire en bloc (hauteur et vitesse d'écoulement importantes).

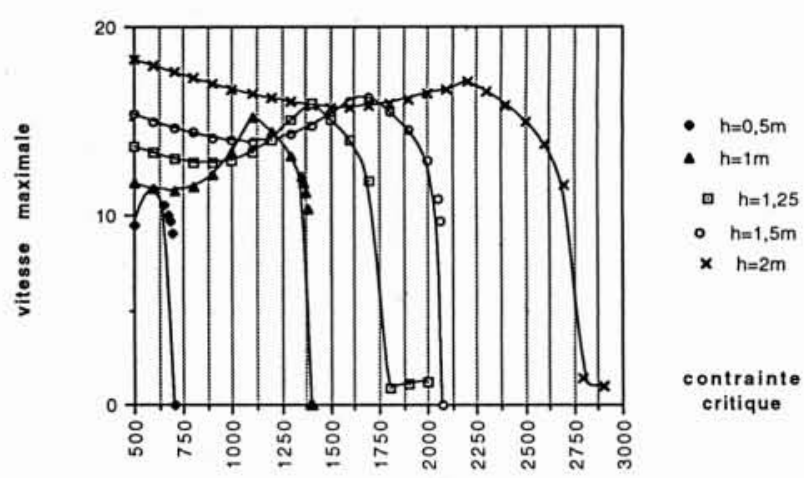

8. Influence de la contrainte critique sur la vitesse maximale de l'avalanche pour différentes hauteurs d'écoulement. 
Cette courbe met aussi en évidence le fait que l'on ne peut pas déterminer de manière unique la contrainte $T_{i}$ en connaissant la vitesse maximale et la hauteur initiale de l'avalanche. Un calage des paramètres dynamiques $(\tau, \mu)$ par la connaissance de l'histoire d'une avalanche est donc rendu malaisé.

\section{Les problèmes annexes}

\subsection{La définition du site}

L'étude d'une avalanche nécessite la définition du site dans lequel elle se produit: le site avalancheux est ainsi la plus petite zone géographique dans laquelle s'écoulent toutes les avalanches quelle que soit leur taille. On effectue ensuite la troncature du site en trois zones indépendantes, comme l'illustre la figure 9

- La zone d'accumulation : c'est le lieu (cirques, grands versants...) sur lequel la neige s'accumule. On divise la zone d'accumulation en panneaux. Chaque panneau est délimité en fonction des conditions de dépôt de la neige et de ses possibilités de décrochement.

- La zone d'écoulement : c'est le lieu par lequel transitent toutes les avalanches majeures du site. Les variations de masse par reprise ou par dépôt y sont négligeables.

- Lazone de dépôt : c'est le lieu sur lequel s'arrêtent toutes les avalanches majeures du site en déposant leur volume de neige transportée.
Le modèle décrit précédemment est centré sur la description de l'avalanche dans la zone d'écoulement et de dépôt. Implicitement, en donnant la condition initiale de neige, on considère que toute la neige de la zone d'accumulation est mise en mouvement, puis transite par la zone d'écoulement. Une telle hypothèse est le plus souvent satisfaisante lorsque l'on s intéresse uniquement de manière grossière aux vitesses atteintes par l'avalanche et aux dimensions de la zone de dépôt. Une description plus fine nécessite de connaître l'écoulement dans la zone d'accumulation ou, de manière artificielle, de fixer un hydrogramme (en fonction du temps) à l'amont de la zone d'écoulement. La suite de ce chapitre présente quelques notions supplémentaires qui peuvent modéliser les phénomènes survenant dans la phase de départ. entre autres, le processus d'incorporation de neige par l'avalanche, que l'on appelle reprise.

\subsection{Stabilité des pentes}

On établit un critère de stabilité en supposant que le manteau neigeux est un ensemble de strates. Chaque couche correspond à un épisode météorologique, a des caractéristiques mécaniques (masse volumique, contrainte critique) qui lui sont inhérentes. et peut évoluer indépendamment du reste du manteau neigeux (différence de tassement, de métamorphisme...). Entre deux strates, on peut trouver une interface de neige sans cohésion (gobelet, givre...) dont la résistance mécanique est nettement plus faible que les autres strates. Ces interfaces sont la cause d'instabilités au sein du manteau qu'elles fragilisent considérablement.

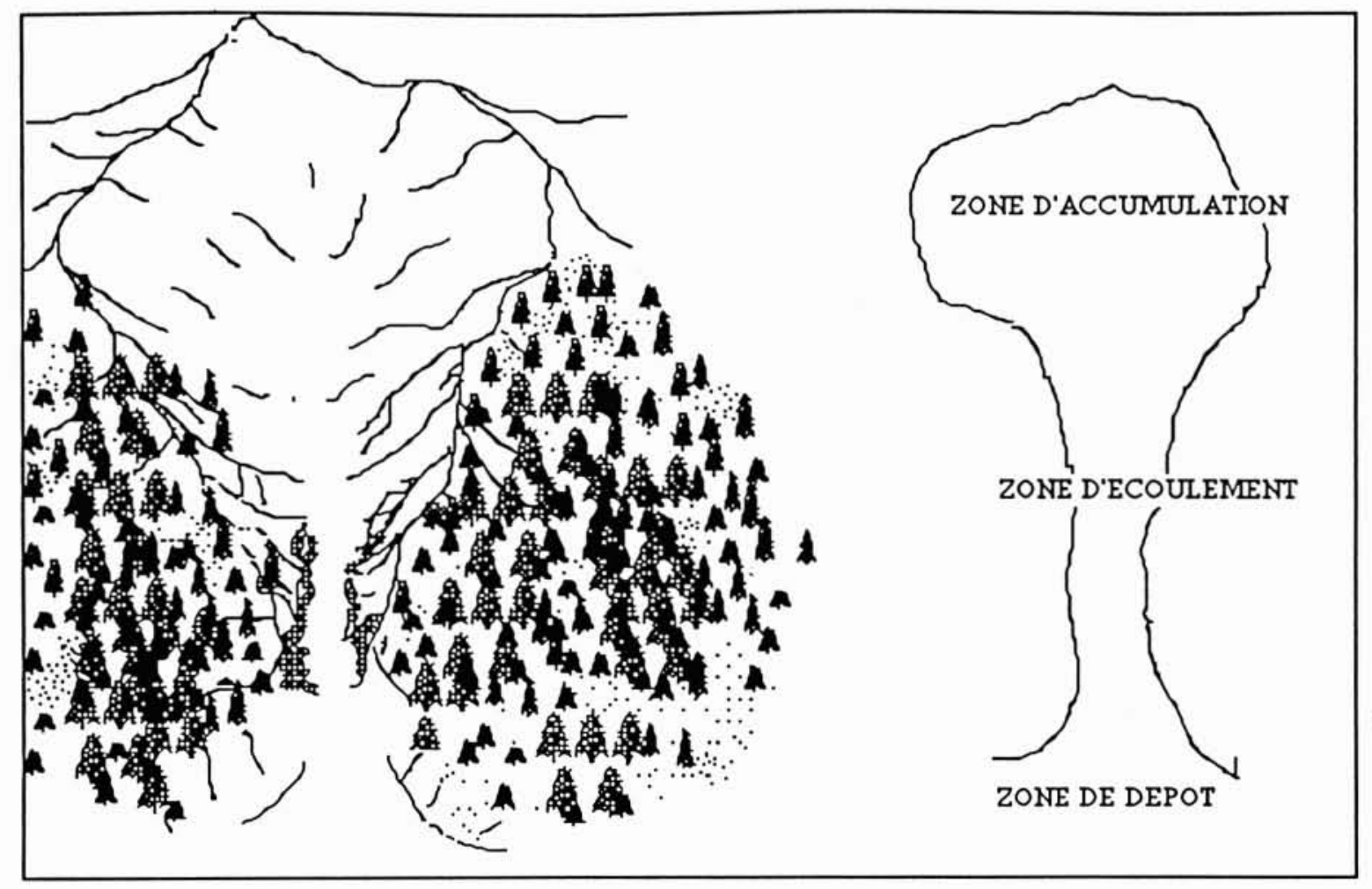

9. Définition des zones du site avalancheux. 


\subsubsection{Définition d'un critère de stabilité}

Le problème de stabilité des pentes est important : il permet d'expliquer la mise en mouvement d'une masse de neige et le phénomène de reprise. L'analyse qui suit est très simple voire simpliste, mais peut éclairer les phénomènes et les quantifier. Il est pour l'instant hors de question de décrire les phénomènes liés au départ de l'avalanche survenant dans la zone d'accumulation, qui sont extrêmement complexes (propagation de contraintes de rupture, grande variabilité des conditions de départ, différents modes de déclenchement) [DuClos, 1993].

L'avantage du modèle de Bingham est qu'il permet grâce à l'existence d'un seuil de contrainte d'introduire une condition nécessaire pour qu'il y ait mouvement; en effet, il est nécessaire que : $\sqrt{-T_{11}}>\tau_{c}$, ce qui dans le cas d'un écoulement stationnaire, simplement cisaillé et unidirectionnel impose :

$$
h>h_{c}=\frac{\tau_{c}}{\rho g \sin \theta} .
$$

On peut supposer qu'un tel critère puisse aussi servir dans le problème de la stabilité du manteau en introduisant alors une contrainte critique statique (et prenant garde de ne pas confondre contraintes statique et dynamique). Une telle analyse, qui s'inspire du critère de rupture de Mohr-Coulomb, a déjà été proposée notamment par [VoITKOVSKI, 1987], qui a tenu compte de la cohésion de la neige. Il introduit un indice de stabilité $K$ défini comme étant le rapport de la contrainte tangentielle limite sur la contrainte exercée, soit :

$$
K(y)=\frac{c(y)}{g \sin \theta \cdot \sum_{y}^{h} \rho_{i} h_{i}}+\frac{f(y)}{\operatorname{tg} \theta}
$$

où $y$ est l'ordonnée, $f$ un terme d'adhérence et $C$ la cohésion. Il y a stabilité si $K>1$. L'équation (45) est similaire en prenant $f=0$, c'est-à-dire que l'on néglige l'adhérence des ancrages du manteau neigeux. On généralise l'équation (45) : la contrainte de cisaillement statique $\tau$ est une fonction croissante, linéaire et continue par morceaux de la profondeur $y$. Le manteau est globalement stable si la répartition des contraintes est toujours comprise dans l'intervalle délimité par les contraintes critiques de chaque couche. La figure 10 illustre cette condition de stabilité. $\mathrm{Si}$ pour une couche $i$ (les couches seront numérotées de haut en bas), on a $\tau>\tau_{i}(i)$, alors il y a rupture de toutes les couches de 1 à $i$.

La figure 10 indique une rupture au niveau de la couche 4 , de faible résistance mécanique (couche de gobelets par exemple). Cette analyse est simple mais peut bien rendre compte de départ en plaque ; des études plus minutieuses ont été réalisées et approfondissent ces notions [FöHN, 1989], [LACKINGER, 1989].

\subsubsection{Données expérimentales sur le critère de stabilité}

L'analyse précédente a déjà été formulée à plusieurs reprises et certains auteurs ont proposé des valeurs des paramètres mécaniques statiques. Entre autres, le Centre d'Etudes de la Neige a réalisé plusieurs campagnes de mesure [BRUN, REY, 1985] et nous donnons maintenant un aperçu sur leurs résultats.

Il faut d'abord prévenir contre l'hétérogénéité des résultats expérimentaux pour une même couche du manteau (dispersion assez importante) [MONTMOLLIN, 1978]. Les paramètres

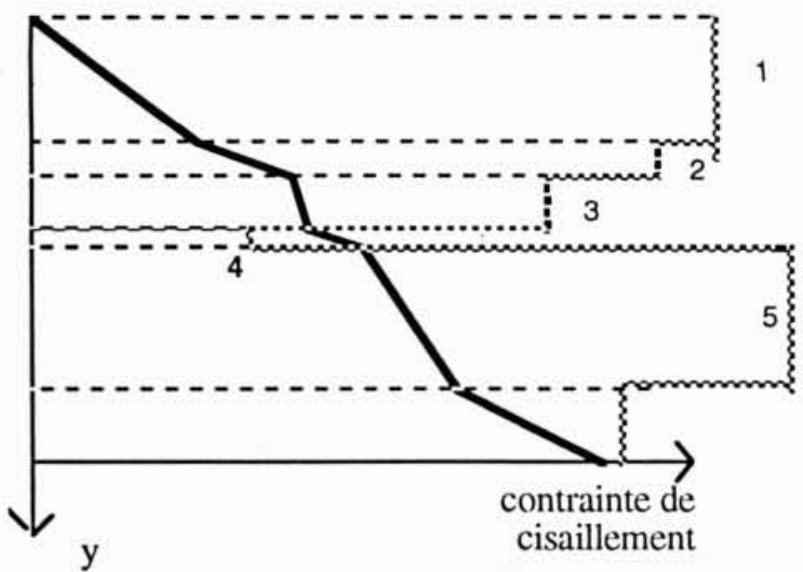

10. Un exemple de répartition des contraintes de cisaillement au sein du manteau neigeux : on indique pour chaque strate la contrainte critique (courbe en pointillé) et la contrainte de cisaillement (courbe continue); pour $i=4$, la contrainte de cisaillement est supérieure à la valeur maximale, il y a rupture.

importants caractérisant la neige sont : sa masse volumique, sa teneur en eau liquide (TEL) et le type de grain.

L'augmentation de la TEL de $0,5 \%$ à $6 \%$ n'entraîne pas de variation de la résistance au cisaillement. Il est à noter qu'une neige mouillée, pour avoir la même résistance qu'une neige sèche, doit avoir une masse volumique plus importante. Il s'ensuit que le passage d'une neige sèche à une neige mouillée induit une baisse importante de la résistance au cisaillement car ce passage se fait sans perte notable de densité. Par la suite, l'augmentation de la TEL n'entraîne que peu d'évolution de la résistance. Une neige normalement drainée ne présente pas de valeur de TEL supérieure à 7 ou $8 \%$. On peut penser toutefois qu ${ }^{\circ}$ une teneur en eau excessive (TEL $>8 \%$ ) est responsable des avalanches de neige mouillée, dont les facteurs sont réunis quand:

- il y a présence d'une couche imperméable (croûte de regel) freinant le drainage de l'eau et favorisant ainsi une forte saturation,

- il y a forte saturation du sol (fonte au printemps),

- il y a des précipitations importantes dépassant la capacité de drainage du manteau.

La TEL trop importante est alors en effet responsable de la disparition des ponts de glace reliant les grains et de la diminution des forces de capillarité (cohésion capillaire).

La résistance statique au cisaillement est augmentée avec la densité (surtout pour des grains fins). On obtient les courbes de corrélation suivantes :

- grains fins (et secs) :

$$
\tau_{i}=0,0981 \cdot \rho^{2.047}-736(\mathrm{~Pa})
$$

corrélation $=0,8$

- neige récente sèche :

$$
\tau_{c}=98,1 \cdot 10^{-6} \rho^{3,239}-72,4(\mathrm{~Pa})
$$

corrélation $=0,91$. 
Ce sont les seuls cas pour lesquels la corrélation est à peu près bonne. La détermination empirique des caractéristiques de la neige est assez malaisée.

\section{Exemple :}

neige à grains fins densité de $200 \mathrm{~kg} / \mathrm{m}^{3}$ : on a alors $\tau_{c}=4300 \mathrm{~Pa}$, la hauteur critique est de $3,1 \mathrm{~m}$ pour une pente à $45^{\circ}$.

\subsection{La reprise}

Après que l'on a exposé la condition de mise en mouvement d'une partie ou de l'ensemble du manteau neigeux, on va exposer comment la reprise est modélisée dans le cas d'un manteau stratifié. Etudions la répartition des contraintes au sein du manteau au-dessus duquel s'écoule une avalanche de hauteur $h_{e}$. A l'interface entre la neige en mouvement et le manteau, la contrainte de cisaillement est $\tau_{f}$. On va supposer que les contraintes réparties dans les strates résultent :

- du poids des strates auquel il faut ajouter la surcharge provoquée par l'avalanche,

- de la force d'entraînement due au mouvement de l'avalanche (qui est l'opposée de la force de frottement selon le principe de l'action et de la réaction).

En ajoutant le poids de l'avalanche, on obtient une courbe translatée de la courbe d'équilibre statique, qui sera de nouveau translatée d'une valeur équivalente à la contrainte de frottement au sol $\tau_{f}$. On obtient finalement pour contrainte totale (en trait gras sur la fig. II):

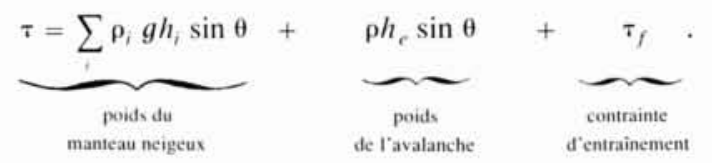

Si, pour une couche donnée, cette contrainte est supérieure à la contrainte critique, c'est la rupture de cette couche, qui entraîne les couches supérieures.

On traduit ainsi la reprise : c'est l'incorporation de la neige dans l'écoulement avalancheux, qui se fait selon l'analyse décrite dans le paragraphe 4.2 par entraînement et surcharge du manteau sous-jacent. La reprise a pour effet d'augmenter la masse de l'avalanche et de réduire sur l'instant sa vitesse.

\section{Conclusions}

Nous avons présenté ici une modélisation des écoulements denses de neige, reposant sur l'intégration des équations du mouvement avec une loi de comportement binghamienne, résolues numériquement par un schéma de Godunov.

Le modèle est dans le principe assez simple et ne nécessite qu'un seul paramètre de calage : le rapport $\tau_{f} / \rho$. L'accent a été mis essentiellement sur l'existence d'un frottement laminaire mais la formulation des équations permet de tenir compte ultérieurement d'un frottement turbulent; ni les données expérimentales, ni les essais numériques n’ont permis de déterminer un critère de transition vers la turbulence.

Le modèle a pour domaine d'application les grosses avalanches denses pour lesquelles l'évolution de la densité n'a que peu d'effet. Le schéma numérique employé est robuste et permet de traiter proprement la discontinuité que constitue le front. Le modèle de Bingham permet de traiter efficacement les conditions d'arrêt et de mise en mouvement de la neige mais si qualitativement, il correspond à des observations que nous avons pu faire sur le terrain, il manque encore crucialement de données expérimentales pour le valider. En dernier lieu, nous avons développé de manière simple certains problèmes annexes comme la stabilité et la reprise.

L'écriture détaillée du modèle a mis en évidence les innombrables problèmes qui surviennent dans la modélisation des avalanches denses, bien que la loi rhéologique choisie demeure très simple et que les hypothèses formulées (écoulement simplement cisaillé et unidirectionnel) simplifient considérablement le problème. Le manque de données expérimentales précises contribue par ailleurs à rendre encore plus délicate la validation de tels modèles, même si les résultats semblent encourageants.

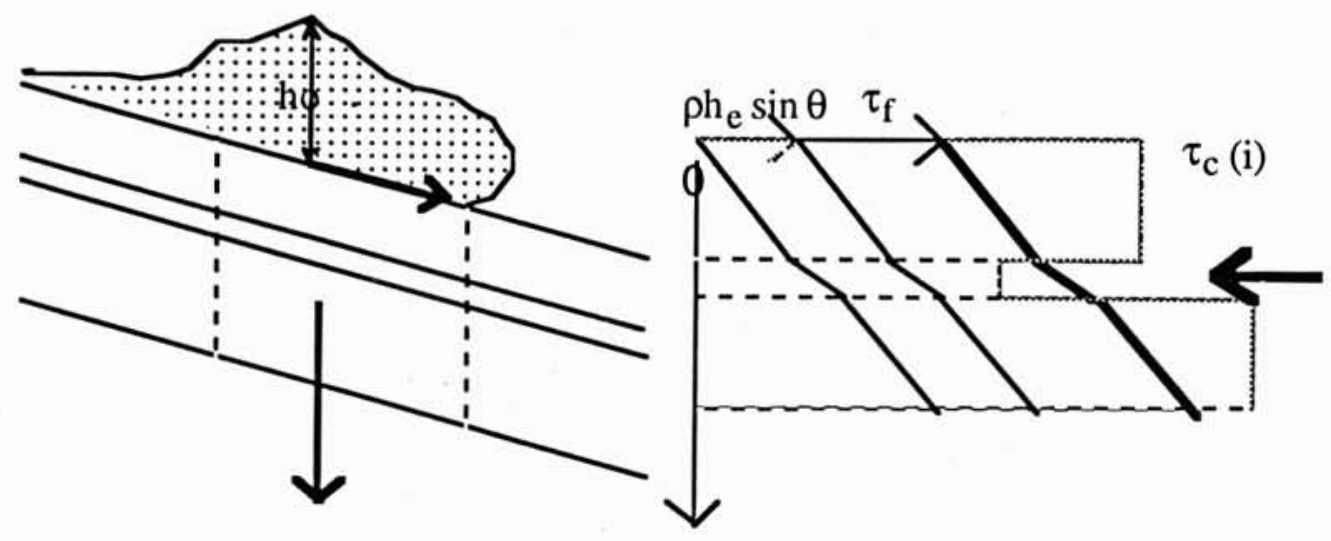

11. Répartition des contraintes au sein du manteau neigeux lors du passage de l'avalanche. La flèche en traits gras indique que la couche intermédiaire cède : il y a alors reprise des 2 couches supérieures par la neige en mouvement. 
Je remercie Mohamed Naaim de la division Nivologie pour son aimable soutien lors des simulations numériques du modèle AVAL DENS et Gérard Brugnod, chef de la division Nivologie qui a soutenu financièrement mon travail. Je sais également gré à Philippe Coussol des critiques constructives qu'il a formulées durant la rédaction de cet article.

\section{Bibliographie}

ANCEY C., 1992. - Modélisations physique et numérique des avalanches denses. Rapport interne CEMAGREFENSHMG.

BAGNOL.D R.A., 1954. - Experiments on agravity free dispersion of large solid spheres in a newtonian fluid under shear. Proceeding of the Royal Society of London, A 225, pp. 49-63.

BÉGHIN P., 1979. - Etudes des bouffées bidimensionnelles. Thèse Joseph Fourier, Grenoble.

Béghin P., Hopfinger E., Britter E., 1981. - Gravitational convection from instantaneous sources on inclined boundaries. Journal of Fluid Mechanics, vol. 107, pp. 407-422.

Béghin P., Olagne X., 1991, - Experimental and theoretical study of the dynamics of powder snow avalanche. Cold Regions Sciences and Technologie, $n^{\circ} 19$.

Bolognesi A., 1991. - L'analyse spatiale des risques d'avalanches. Thèse Université Joseph Fourier, Grenoble.

BRUN E., REY L. - Bilan de la campagne de mesures mécaniques de la neige effectuées sur le terrain durant l'hiver 1984-1985. Centre d'Etude de la Neige, Grenoble, Notes de travail. $n^{\circ} 199$

Buggisch H., Stadler R., 1986. - On the relation between shear rate and stresses in one-dimensional steady flow of moist bulk solids. Proceedings of the World Congress Particle Technology, Part III, Mechanics of Pneumatic and Hydraulic conveying and Mixing, Nürnberg. pp. 187-202,

Buisson L., Charlier C., 1989. - Avalanche starting-zone analysis by use of knowledge-based system. Annals of Glaciology $n^{\circ} 13$, pp. 27-30.

BUSER O., 1989. - Two years experience of operational avalanche forecasting using the nearest neighbours method. Annals of Glaciology, $\mathrm{n}^{*} 13$, pp. 31-34.

CARrY C., POCHAT R. - Modélisation d'une avalanche Compte-rendus de la $2^{\mathrm{c}}$ rencontre internationale sur la neige et les avalanches, 12, 13 et 14 avril 1978, pp. 189 . 221.

DENT J., 1980. - A biviscous modified Bingham model of snow avalanche motion. Ph. D. thesis, Montana State University, Bozeman, Montana.

DENT J., LANG T.E., 1983. - A biviscous modified Bingham model of snow avalanche motion. Annals of Glaciology, vol. 4 , pp. 131-140.

Duclos A., 1993. - Contribution à l'étude de la localisation des plaques de neige sur un domaine skiable : conception d'un protocole d'observations et exploitation des résultats de la saison 1992-1993 pour une station de HauteMaurienne. Mémoire de DEA (IGA), université Joseph Fourier.

EgLIT M.E., 1984. - Theoretical Approches to avalanche dynamics. Soviet Research Avalanche Biography update 1977-1983, Glaciological data, Report GD-16, US department of Agriculture Forest Service, World Data Center for Glaciology, pp. 63-117.
Fön P.M.В., 1987. - The stability index and various trigerring mechanisms. Proceedings of Symposium of Davos 1986, IAHS, n 162, pp. 195-241.

GüBler H., 1989. - Comparison of three models of avalanche dynamics. Annals of glaciology, $\mathrm{n}^{\circ} 13$, pp. 82-89.

HANKS R.W. and DAHIA B.H., 1971. - Theoretical analysis of the turbulent flow of non-newtonian slurries in pipes. American. Industrial. Chemical Engineering. Journal, $\mathrm{n}^{\circ} 17$, pp. 554-557.

HOPFINGER E., 1983. - Snow avalanche motion and related phenomena. Annals of Fluid Mechanics, n*15, pp. 4776.

HUTTER K., 1986. - Gramular shear flows as models for flow avalanches. Symposium de Davos 1986, IAHS n*162. pp. 381-394.

HUTTER K., 1989. - Numerical, analytical and laboratory experimental studies of granular avalanche flows. Annals of Glaciology, vol. 13, pp. 109-116.

LACKINGER B., 1989. - Supporting forces and stability of snow-slab avalanches, a parameter study. Annals of Glaciology, $\mathrm{n}^{\circ} 13$, pp. 140-145.

LANG T.E., BRown R.L., 1980, - Numerical solution of snow avalanche impact on structures. Research Report RM216 USDA-Rocky Mountain Forest and Rang Experiment Station Ft Collins USA.

LIED K., TOPPE R., 1989. - Calculation of maximum snow avalanche run-out distance by use of digital terrain models. Annals of Glaciology, n'13, pp. 164-169.

LUMLEY J.L., 1976. - Two phase and non-newtonian flows. Topics in Applied Physics, vol. 12, Turbulence, édité par P. Bradshaw Springer New York, pp. 290-324.

MAC Clung D.M., LIED K., 1987. - Statistical avalanche zoning. Proceedings of the symposium on avalanche formation, movement and effects. IAHS Publication n" 162 .

MAEno N., Nishimura K., 1989. - Contribution of viscous forces to avalanche dynamics. Annals of Glaciology, vol. 13, pp. 202-205.

Marco O., 1993. - Mesures des paramètres physiques dans un écoulement avalancheux. Thèse Université Joseph Fourier en préparation, Grenoble.

Martinelli M., Lang T.E., Mears A., 1980. - Calculation. of avalanche friction coefficients from field data. Journal of Glaciology, vol. 26, $n^{\circ} 94$.

MARTINET G. - Contribution à la modélisation numérique des avalanches de neige dense et des laves torrentielles. Thèse Université Joseph Fourier, CEMAGREF. Grenoble 1992, non publiée.

Mıdoux N., 1985. - Mécanique et Rhéologie des fluides en génie chimique. Tec \& Doc Lavoisier, Paris.

Montmollin V., 1978. - Introduction à la Rhéologie de la neige. Thèse Université Joseph Fourier, Grenoble.

NOREM H., 1986. - A continuum model for calculating snow avalanche and velocities. NGI Report and Proceedings of the symposium on avalanche formation Davos 1986. IAHS $n^{\circ} 162$

NOREM H., 1989. - Simulation of snow avalanche in run-out zones. Annals of Glaciology, $\mathrm{n}^{\circ} 13$, pp. 218-225.

NOREM H., 1992. - Simulation of snow avalanche flow by a continuum granular model. Compte-rendu de l'université d'été de Chamonix ANENA.

PERLA R. et al., 1981. - On the Computation of parameters that model snow avalanche motion. Canadian Geotechnical Journal 18, 121, pp. 1-10. 
Piau J.M., 1979. - Les fluides non newtoniens. Techniques de I’Ingénieur, A 710-711.

SALM B., BURKARd A., GUBler H., 1990, - Berechnung von Fliesslawinen, eine Anleitung für Praktiker mit Beispielen. Eigdenössichen Institut für Schnee- und Lawinenforschung. $n^{*} 47$ (traduction française dans Ancey).

SAVAGE S.B., 1984. - The mechanics of rapid granular flow: Advances in Applied Mechanics, vol. 24. pp. 289-366.

SAVAGE S.B., HUTtER K.. 1991, - The dynamics of avalanches of gramular materials from initiation to runout. Part I: Analysis. Acta Mechanica 86. 1991. Springer-Verlag. pp. 201-223.

SAvAge S.B., Hutter K., 1989. - The motion of a finite mass of gramular material don'n a rough incline. Journal of Fluid Mechanics, vol. 199, pp. 177-215.

Savage S.B., Sayed M., 1984. - Stresses developed by dry cohesionless granular materials in an annular shear cell. Journal of Fluid Mechanics, $n^{*}$ 142. pp. 391-430.

STADL.ER R.. BUGgish H.. 1985. - Influence of the deformation rate on shear stress in bulk solids, theoretical aspects and experimental results. EFCE Publications series $n^{\circ} 49$, Reliable flow of particulate Solids, Proceedings Bergen, Norway, p. 15.

Trowbridge J.H., 1987. - Instability of concentrated free surface flows. Journal of Physical Research, vol. 92. n" C9. pp. 9523-9530.

UNESCO, 1981. - Avalanche Atlas. Commission Internationale sur la Neige et la Glace de I'IAHS (responsable De Quervain).

VILA J.P., 1986. - Sur la théorie et l'approximation numérique des problèmes hyperboliques non linéaires. application aux équations de Saint Venant et à la modélisation des avalanches de neige dense. Thèse Université Paris VI.

Vil.A J.P., DuBoIS F., ChARGY D., 1992. - Méthodes numériques pour le calcul d'écoulements compressibles. Institut pour la promotion des sciences de l'ingénieur. Paris.

VOELLMY A., 1955. - Ober die Zerstörungskraft von Lawinen. Schweizerische Bauzeitung 73, vol. 12, pp. 159-162.

Vortkovskı K.F.. 1987. - Snow cover stahility on slopes and avalanche dynamics. Proceedings of Symposium of Davos 1986, IAHS n" 162, pp. 337-351. 